\title{
Extended midpoint method for solving fuzzy differential equations
}

\begin{abstract}
We study fuzzy differential equations (FDEs) using the strongly generalized differentiability concept. Utilizing the characterization problem, we present approximate solutions of FDEs under Generalized differentiability by an equivalent system of ODEs. Then we extend midpoint approximation method and give its error, which guarantees pointwise convergence. An illustrative example is given.
\end{abstract}

Keyword: Fuzzy differential equations; Generalized differentiability; Generalized characterization theorem; Midpoint method 